Mater. Res. Soc. Symp. Proc. Vol. 1344 (C) 2011 Materials Research Society

DOI: 10.1557/opl.2011.1347

\title{
Self-aligned Graphene Sheets-Polyurethane Nanocomposites
}

Mohsen Moazzami Gudarzi ${ }^{1,2}$, Seyed Hamed Aboutalebi ${ }^{1}$, Nariman Yousefi ${ }^{1}$, Qing Bin Zheng ${ }^{1}$, Farhad Sharif ${ }^{2}$, Jie $\mathrm{Cao}^{3}$, Yayun $\mathrm{Liu}^{3}$, Allison Xiao ${ }^{3}$ and Jang-Kyo Kim ${ }^{1 *}$

${ }^{1}$ Department of Mechanical Engineering, Hong Kong University of Science and Technology, Clear Water Bay, Kowloon, Hong Kong

${ }^{2}$ Department of Polymer Engineering, Amirkabir University of Technology, 424 Hafez Ave, Tehran, Iran

${ }^{3}$ Advanced Technologies, Henkel Corporation, 10 Finderne Ave. Bridgewater, NJ 08807, USA

\begin{abstract}
Processing graphene and graphene polymer nanocomposites in an aqueous medium has always been a big challenge due to the hydrophobic nature of graphene (or reduced graphene oxide) nanosheets. In this work, a waterborne latex of polyurethane has been used both as the matrix material for embedding the graphene nanosheets and as a unique stabilizer to help produce an up to $5 \mathrm{wt} \%$ graphene/PU nanocomposites. The graphene oxide/polyurethane latex aqueous suspension is reduced in-situ using hydrazine, without any trace of aggregation/agglomeration upon completion of the reduction process, which would otherwise have occurred severely were PU not present. A highly aligned nanostructure is produced when graphene content is increased beyond $2 \mathrm{wt} \%$, resulting in a remarkable improvement in electrical and mechanical properties of the nanocomposite. The exceptionally low electrical percolation threshold of $0.078 \%$, as well as 21-fold and 14 fold increases in tensile modulus and strength, respectively, have been attained thanks to the alignment of graphene nanosheets in the polymeric matrix.
\end{abstract}

\section{INTRODUCTION}

Graphene, a single atomic layer of $\mathrm{sp}^{2}$ carbon atoms has gained much attention in recent years. This unique arrangement of carbon atoms bring about exclusive electrical, thermal, magnetic and mechanical properties [1-4]. Graphene is known to be the hardest and stiffest material on earth. What adds up to these fascinating properties of graphene is the mere fact that it can be produced from cheap and naturally occurring graphite flakes [5-7]. Being one of the thinnest and strongest materials with exceptionally high electron mobility and thermal conductivity suggests that one of the most efficient ways to benefit from the properties of graphene is to incorporate them in a polymeric material to form nanocomposites [8]. Therefore, graphene oxide $(\mathrm{GO})$ and reduced graphene oxide ( $\mathrm{rGO}$ ) sheets have attracted significant attention as filler for polymer nanocomposites that are now finding diverse applications [9-11]. 
This paper presents a simple method for the production of polymer-ultra-large graphene composites using water-borne polyurethane (PU) latex where the polymer serves both as stabilizer in the reduction step as well as the matrix material. Interparticle interactions between the ultra-large size GO sheets and PU particles are considered as the key underlying mechanism for the stabilization. The unique morphology of PU-graphene dispersion resulted in self-alignment of GO sheets with strong interfacial interactions with the matrix. The resulting composites exhibited exceptional mechanical properties and electrical conductivities with one of the lowest percolation threshold values ever reported.

\section{EXPERIMENTAL}

Ultra-large size GO (UL-GO) was synthesized based on the modified chemical method [7] using expanded graphite. The obtained GO particles were diluted using DI water $(\sim 1 \mathrm{mg} / \mathrm{ml})$ and mildly sonicated to avoid breakage. The diameter of the as-produced GO sheets ranged between sub-micron to two hundred $\mu \mathrm{m}$, with an average size of $32.7 \pm 24.3 \mu \mathrm{m}$ [12]. The GO dispersion was mixed with aqueous emulsion of polyurethane (PU) to obtain a homogeneous aqueous dispersion. Hydrazine solution was added in the weight ratio of 3:1 to obtain reduced $\mathrm{GO}(\mathrm{rGO})$, which was then heat-treated at $80^{\circ} \mathrm{C}$ for $24 \mathrm{hr}$. The mixture was poured into a flat mold and dried in an oven at $50^{\circ} \mathrm{C}$ for $6 \mathrm{hr}$ to produce composite films. The final products were highly flexible, black composite films.

Zeta potentials analyzer (Brookhaven ZetaPlus), transmission electron microscopy (TEM, JEOL 100X), scanning electron microscope (SEM, JEOL 6390F), tapping-mode atomic force microscope (AFM, Digital Instruments), four-point probe method using a resistivity/Hall measurement system (Scientific Equipment \& Services) and dynamic mechanical analyzer (DMA 7, Perkin Elmer) were used to characterize the samples.

\section{DISCUSSION}

\section{$\underline{\text { Stabilization and alignment of rGO using polyurethane dispersion }}$}

As mentioned in the experimental section, the nanocomposites were prepared by hydrazine reduction of PU/GO colloidal mixture. The reduced mixture maintained a remarkable stability, though rGO was highly hydrophobic. This phenomenon can be explained in light of the surface charge of the colloidal particles. Zeta potential is a measure of the surface electrical charges and the stability of the colloidal dispersions. The Zeta potentials of PU, GO, rGO and PU/GO colloidal mixtures have been measured and presented in Figure 1. The addition of PU to GO further increases $|\xi|$, therefore addition of PU latex particles has a pronounced effect on stabilizing GO. Figure 2 shows a photograph of GO, rGO and PU/GO dispersions one month 
after their initial synthesis. It can be seen that rGO was fully agglomerated but the samples containing the mixture of $\mathrm{PU} / \mathrm{rGO}$ and $\mathrm{GO}$ alone were perfectly stable.

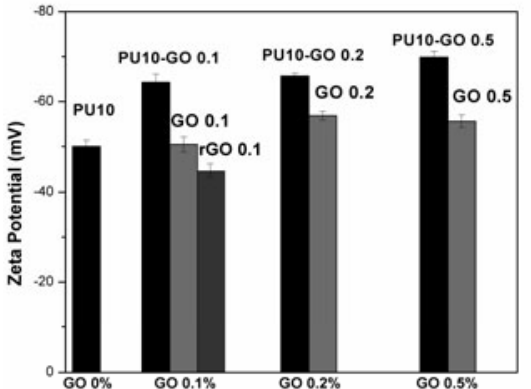

Figure 1. Zeta potentials of $\mathrm{PU}, \mathrm{GO}, \mathrm{PU} / \mathrm{GO}$ and $\mathrm{PU} / \mathrm{rGO}$ hybrid dispersions.

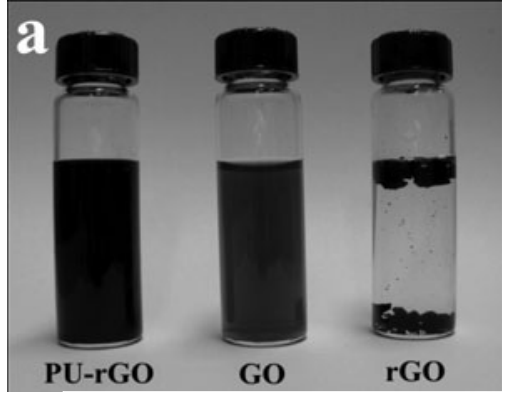

Figure 2. Photographs of GO, rGO and $\mathrm{PU} / \mathrm{rGO}$ dispersions.

It is thought that polymer particles adhere to the graphene surface and fuse together to form a uniform polymeric coating on the GO sheet surfaces, which is mainly responsible for attributing the stability of $\mathrm{PU} / \mathrm{rGO}$ colloids. Alike macromolecules, polymer particles can also be adsorbed onto the surface of GO sheets [13].This process is perhaps enthalpically favorable because of possible interaction between the hydrophilic groups on the surface of both the GO and PU particles [14].

The formation of the mentioned polymeric coating layer can also be seen in AFM and TEM images. Figures $3 \mathrm{a}$ and $3 \mathrm{~b}$ depict the typical AFM scans of graphene and PU covered graphene, respectively. The height profile is included for a more quantitative treatment. It can be seen that the average step height of GO was around 0.7-1.0 nm which has been well documented as the thickness of a single layer GO sheet [5]. Interestingly, when PU is introduced to GO this height changes to $4.0-5.0 \mathrm{~nm}$, as a result of adherence and fusing of latex particles onto the GO surface which lead to the formation of a uniform polymeric film.

TEM studies (Figures $3 \mathrm{c}$ and $3 \mathrm{~d}$ ) also support the proposed mechanism. A comparison between GO (figure 3c) and PU/rGO (figure 3d) indicated an increase in contrast when PU was introduced, which can be interpreted as an increase in the layer thickness, consistent with the AFM micrographs. The uniform nature of the formed film can be better seen in TEM pictures. Therefore, it can be said that the latex particles fuse together to form a stable and uniform film.

The freeze fracture surface of the nanocomposites have been studied using SEM. Figure 4 shows the typical micrographs for nanocomposites containing up to $5 \mathrm{wt} \%$ of GO sheets. The graphene sheets were seen well dispersed and no trace of aggregation/agglomeration was found 
in the typical fracture surfaces, given the fact that no graphene sheet was directly exposed to the fracture surface $[15,16]$. This observation reflects strong interactions and adhesion between the polar PU macromolecules and the oxygenated functionalities available on the partially reduced GO sheets.

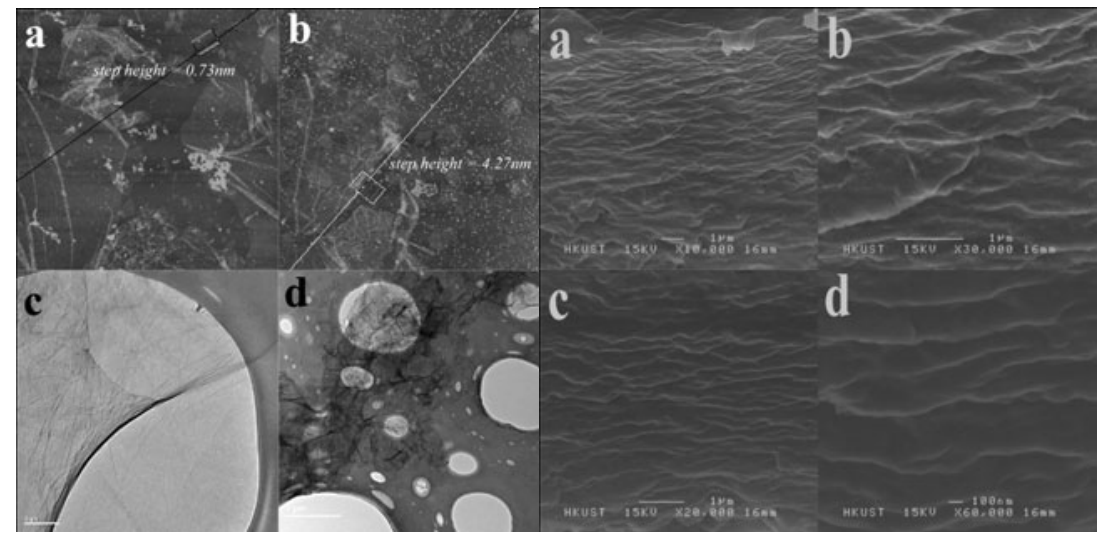

Figure 3. AFM images of a) GO and b) PU/rGO Figure 4. SEM images of freeze fracture surface of composite. TEM micrographs of c) GO and d) PU/rGO composites containing a,b) $2 \mathrm{wt} \%$ and c,d) $\mathrm{PU} / \mathrm{rGO}$. The scale bar in $\mathrm{c}, \mathrm{d})$ is $1 \mu \mathrm{m}$. $5 \mathrm{wt} \%$ graphene.

A more interesting feature associated with the fracture surfaces is the aligned nature of $\mathrm{GO}$ sheets, specifically in the highly-filled $5 \mathrm{wt} \% \mathrm{PU} / \mathrm{rGO}$ sample. The degree of alignment increased with increasing graphene content, therefore the UL-GO sheets tend to self-align upon evaporation of water. This is a possible scenario as a result of the very large aspect ratio of the GO sheets, approximately 30000 [12], and the low viscosity of the medium (water) both of which facilitates the mobility of nanosheets. In fact, the steric hindrance arising between the microscopically large graphene sheets of a high aspect ratio induced rearrangement into a layered structure during drying, while the low viscosity medium, such as water, and the long drying time encouraged the near-perfect alignment of graphene sheets.

\section{Electrical conductivity}

The high electrical conductivity and high aspect ratio of graphene make it an outstanding candidate for applications to avoid electrostatic and magnetic interference. The electrical conductivity versus graphene content of PU/rGO nanocomposites is presented in Figure 5 . The percolation threshold of this system seems(see inset of Figure 5) to be as low as $0.078 \mathrm{wt} \%$, 
which, to of our knowledge, is the lowest value reported for homogeneous polymer-graphene nanocomposites in the open literature. This exceptionally low value can be attributed to the highly-aligned monolayer graphene sheets with an extremely large aspect ratio as a result of the unique processing method developed in this study.

\section{Mechanical properties of $\mathrm{PU} / \mathrm{rGO}$ composites}

Graphene is the strongest material ever synthesized [6], therefore, it can be used to enhance the mechanical properties of polymers. The tensile modulus and strength of neat PU and the corresponding nanocomposites are presented in Figure 6. In is noteworthy to mention that addition of only $0.3 \mathrm{wt} \%$ of graphene increased the tensile modulus and strength of the nanocomposites by $110 \%$ and $390 \%$, respectively. When the graphene content was further raised to $3 \mathrm{wt} \%$, remarkable 21 -fold and 14 -fold increases were achieved.

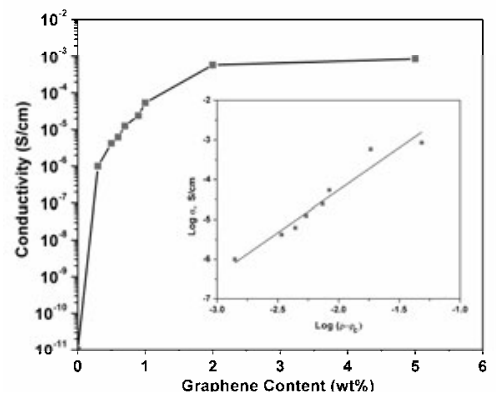

Figure 5. Electrical conductivity of PU-rGO composites as a function of graphene content, $\rho$.

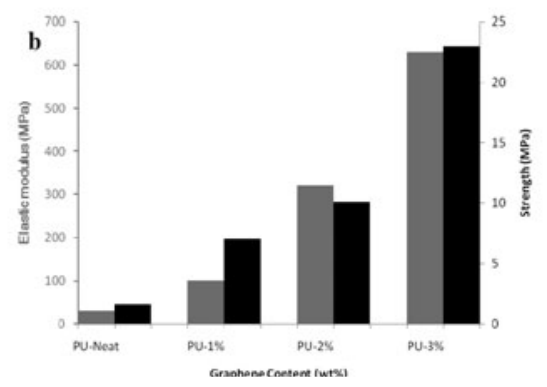

Figure 6. Mechanical properties of PU-rGO composites as a function of graphene content.

Such remarkable improvements in mechanical properties are attributed to three interrelated factors, namely i) fine exfoliation of graphite nanoplatelets into ultra-large size, monolayer graphene sheets with high aspect ratios; ii) self-alignment of individual graphene sheets when the graphene content is above a threshold value; and iii) strong interfacial interaction between the graphene sheets and PU matrix.

\section{CONCLUSION}

Waterborne PU latex was used to produce nanocomposites containing well dispersed and highly oriented UL-GO sheets. PU not only functioned as a continuous matrix for embedding the GO sheets, but also stabilized the extremely hydrophobic rGO in the aqueous medium. It was shown that when the graphene content exceeded $2 \mathrm{wt} \%$, a highly aligned layered structure was formed which mimics that of the naturally occurring nacre shells. Remarkable 21 -fold and 14-fold 
increases in the tensile modulus and strength, respectively, were achieved by the addition of $3 \mathrm{wt} \%$ of graphene. The composites also showed excellent electrical conductivity with an exceptionally low percolation threshold of $0.078 \mathrm{vol} \%$, which is one of the lowest values reported in the literature for polymers containing graphene.

\section{REFERENCES}

[1] K. S. Novoselov, A. K. Geim, S. V. Morozov, D. Jiang, Y. Zhang, S. V. Dubonos, I. V. Grigorievaa and A. A. Firsov, Science 306, 666.

[2] C. N. R. Rao, A. K. Sood, K. S. Subrahmanyam and A. Govindaraj. Angew. Chem. Int. Ed. 48, 7752 .

[3] A. K. Geim. Science 324, 1530 (2009).

[4] C. Soldano, A. Mahmood and E. Dujardin. Carbon 48, 2127 (2010).

[5] D. R. Dreyer, S. Park, C. W. Bielawski and R. S. Ruoff. Chem. Soc. Rev. 39, 228 (2010).

[6] S. Park and R. S. Ruoff. Nat. Nanotech. 4, 217 (2009).

[7] Y. Geng, S. J. Wang and J. K. Kim. J. Colloid Interf. Sci. 336, 592 (2009).

[8] S. Stankovich, D. A. Dikin, G. H. B. Dommett, K. M. Kohlhaas, E. J. Zimney, E. A. Stach, R. D. Piner, S. T. Nguyen and R. S. Ruoff. Nature 442, 282 (2006).

[9] T. Ramanathan, A. A. Abdala, S. Stankovich, D. A. Dikin, A. M. Herrera, R. D. Piner, D. H. Adamson, H. C. Schniepp, X. Chen, R. S. Ruoff, S. T. Nguyen, I. A. Aksay, R. K. Prud'Homme and L. C. Brinson. Nat. Nanotechnol. 36, 327 (2008).

[10] H. Kim, A. A. Abdala and C. W. Macosko. Macromolecules 43, 6515 (2010).

[11] D. Cai and M. Song. J. Mater. Chem. 20, 7906 (2010).

[12] S. H. Aboutalebi, M. M. Gudarzi, Q. B. Zheng and J. K. Kim, Adv. Funct. Mater. in press (2011).

[13] M. M. Gudarzi and F. Sharif. J. Colloid Inter. Sci. 349, 63 (2010).

[14] D. K. Chattopadhyay and K. V. S. N. Raju. Prog. Polym. Sci. 32, 352 (2007).

[15] J. Li, L. Vaisman, G. Marom and J. K. Kim. Carbon 45, 744 (2007).

[16] J. Li, J. K. Kim, M. L. Sham and G. Marom. Compos. Sci. Technol. 67, 296 (2007) 